\title{
Calculation of Onset Voltage of Sliding Discharge over a Dielectric Barrier
}

\author{
Mazen Abdel-Salam ${ }^{1, a}$, Azza Hashem ${ }^{2}$ and Hadeer El-Hawary ${ }^{2}$ \\ ${ }^{1}$ Department of Electrical Engineering, 71516, Assiut University, Assiut, Egypt. \\ ${ }^{2}$ Department of Physics, 71516, Assiut University, Assiut, Egypt.
}

\begin{abstract}
This paper is aimed at calculating the onset voltage of a sliding discharge established between two electrodes at the upper and bottom surfaces of a dielectric barrier plate; named sliding dielectric barrier discharge (SDBD) driven by AC voltage during negative half cycle. The onset voltage is based on the condition of self-sustenance of avalanche growth in the vicinity of the stressed electrode. This calls at first for calculation of the electric field in the vicinity of stressed electrode. The dependency of onset voltage on the thickness and permittivity of the dielectric barrier as well as the thickness of the stressed electrode and the inter-electrode spacing between the stressed and ground electrodes is investigated. The obtained results are discussed in the light of gas discharge physics.
\end{abstract}

\section{Introduction}

The sliding discharge over a dielectric barrier (SDBD) has been studied for more than fifteen years to harness it for use in electro hydrodynamic actuators. Such discharge is generated on the surface of a dielectric plate sandwiched between upper and bottom electrodes. The upper electrode is stressed by DC and AC voltages with respect to the bottom one [1] with a focus on the discharge current pulses.

The velocity induced by the plasma actuator and dissipatedpower were recorded as a function of the applied voltage [2,3]. A numerical modeling of a single micro discharge together with experimental finding made it possible to develop a simplified analytical model of the body force induced by sliding discharge driven by AC voltage [3, 4]. All those studies [1-4] did not report on the assessment of the onset voltage of sliding (or dielectric barrier) discharge SDBD.

This paper is aimed at calculating the onset voltage of sliding discharge over a dielectric barrier based on the condition of self-sustenance of avalanche growth within the ionization zone around the AC stressed electrode during negative half-cycle. This calls at first for calculation the electric field in the vicinity of stressed electrode. The dependency of onset voltage on the thickness and permittivity of the dielectric barrier as well as the thickness of the stressed electrode and the inter-electrode spacing between the stressed and ground electrodes is investigated. The obtained results are discussed in the light of gas discharge physics.

\section{Method of Analysis}

\footnotetext{
${ }^{\mathrm{a}}$ Corresponding author : mazen2000as@yahoo.com 


\subsection{Electric field calculation}

The calculation of the onset voltage calls for electric field assessment at the barrier surface using the charge simulation method.

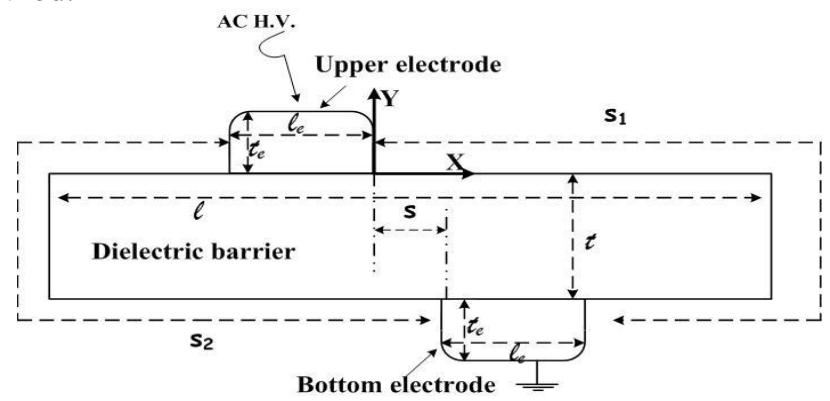

Figure 1. Upper and bottom electrodes sandwiching a dielectric barrier

\subsubsection{Electrodes and dielectric arrangement for SDBD}

Figure 1 shows a cross-section as viewed in the $x-y$ plane of an electrode arrangement selected to assess the onset voltage of sliding discharge over a dielectric plate. The electrode arrangement is considered long along the $\mathrm{z}$-direction. This electrode arrangement was selected before to investigate the surface dielectric barrier discharge (SDBD) [1]. The dielectric plate with width $l$, thickness $t$ and permittivity $\varepsilon_{r}$ extends between upper and bottom electrodes as shown in Fig. 1. The width and thickness of both the upper and bottom electrodes are $l_{e}$ and $t_{e}$, respectively. The spacing $S$ between the front edge of the upper electrode and the back edge of the bottom one is variable by moving the bottom electrode with respect the upper one. The distance $S_{1}$ over the dielectric surface is longer than $S_{2}$ to ensure the occurrence of the sliding discharge over $S_{1}$. The upper electrode is stressed by AC voltage with respect to the bottom one. Therefore, the two electrodes are charged provided that the dielectric plate and the surrounding air are perfect dielectrics.

\subsubsection{Charge simulation of electrodes and dielectric barrier}

With the applied voltage, each charged electrode is simulated by a set $N_{e}$ of line charges extending parallel to its axis. Fig. 2. The interface between two dielectrics (dielectric plate and air) is simulated by two sets of line charges, each of number $N\left(=N_{a}=N_{d}\right)$, one in the dielectric plate and the other in the air, extending parallel to the axis of the dielectric plate, Fig. 2. Therefore, the total number of the line charges simulating the upper and bottom electrode as well as the dielectric plate is $2 N_{e}+2 N$.

A set of $2 N_{e}+2 N$ simulations equations is to be formulated and solved to determine the unknown simulation charges. This set is to satisfy pertinent boundary conditions at boundary points selected over the boundaries ofFig. 1. Boundary points of number $N_{e}$ are selected over the surface of each electrode inFig. 2, so each boundary point corresponds to a simulation charge in the electrode. The distance $d_{1}$ is chosen equal to the distance $d_{2}$ as shown in Fig 3 (a). Also, $N$ boundary points are selected over the periphery of the interface between the dielectric plate and the surrounding air inFig. 2 , so each boundary point corresponds to two simulation charges, one in the air and the other in the dielectric plate. The distance $d_{3}$ between two successive boundary points on the interface is equal to $d_{4}\left(=d_{5}\right)$ provided that $d_{4}$ and $d_{5}$ are the distances between the boundary point and the corresponding simulation charges in air and dielectric plate, respectively as shown in Fig. 3 (b). With the determination of the simulation charges, the electric potential and field can be calculated anywhere around the electrodes and the dielectric barrier. 


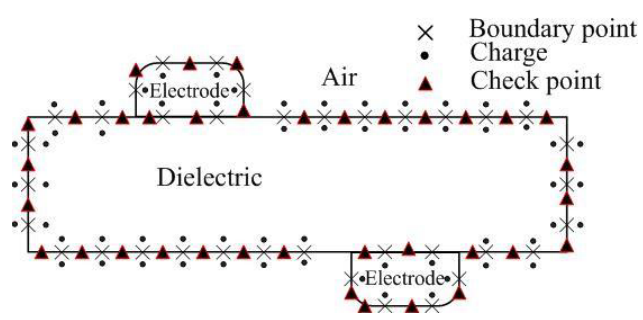

Figure 2. Simulation charge arrangement for the electrodes and dielectric barrier.

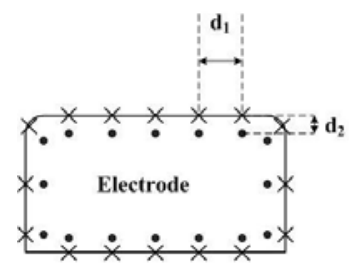

(a)

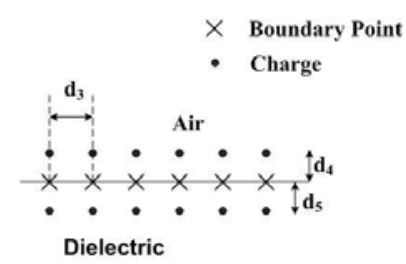

(b)

Figure 3. Boundary points arrangement at the electrode bound and air-dielectric interface.

\subsubsection{Pertinent boundary conditions}

The boundary conditions are Dirichlet condition at the electrodes' surface where the calculated potential is equal to the applied voltage as well as the equality of air-side and dielectric-side potentials and Neumann condition at the interface between the dielectric plate and surrounding air, where the calculated normal electric flux is continuous.

\subsubsection{Describing equations}

For the $\mathrm{j}^{\text {th }}$ simulation charge $q_{j}$ at $\left(x_{j}, y_{j}\right)$, the potential at point $P_{i}$ with $\left(a_{i}, b_{i}\right)$ is expressed as

$$
\emptyset(i, j)=P_{i j} q_{j}
$$

where $P_{i j}$ is the potential coefficient due to the $\mathrm{j}^{\text {th }}$ simulation charge at the $\mathrm{i}^{\text {th }}$ point as expressed as

$$
P(i, j)=\frac{1}{4 \pi \varepsilon_{\circ}} \frac{1}{r_{i j}}, \quad r_{i j}=\left[\left(x_{j}-a_{i}\right)^{2}+\left(y_{j}-b_{i}\right)^{2}\right]^{0.5}
$$

where $\varepsilon_{\circ}$ is the permittivity of the space.

The $x$ and $y$ components of the electric field are expressed as

$$
E_{x}(i, j)=F_{x}(i, j) q_{j}, \quad E_{y}(i, j)=F_{y}(i, j) q_{j}
$$

where $F_{x}(i, j)$ and $F_{y}(i, j)$ are $x$ - and $y$ - field coefficient due to the $j^{\text {th }}$ simulation charge at the $i^{\text {th }}$ point along $x$ - and $y$-directions,

$$
F_{x}(i, j)=\frac{1}{4 \pi \varepsilon \circ} \frac{x-a_{i}}{r_{i j}{ }^{3}}, \quad F_{y}(i, j)=\frac{1}{4 \pi \varepsilon \circ} \frac{y-b_{i}}{r_{i j}{ }^{3}}
$$

Satisfaction of the above mentioned boundary conditions at the selected boundary points formulates a set of equations, whose simultaneous solution of this set of equations determines the unknown simulation charges.

\subsubsection{Accuracy of charge simulation methodology}

To check the accuracy of the chosen charge simulation, check points have been located midway between the boundary points. Satisfaction of the above-mentioned boundary conditions at the check points is a measure of the solution accuracy.

\subsection{Calculation of onset voltage of SDBD.}


When the instantaneous value of the electric field at the stressed electrode during the negative half cycle of the applied voltagereaches the threshold value for ionization by electron collision, an electron avalanche starts its growth. The avalanche extends along the maximum electric field stream line in the ionization zone surrounding the stressed electrode. The number of electrons at a distance $x$ from the starting point of the avalanche growth, Fig. 4 is expressed as:

$$
N_{e}(x)=\gamma_{p h} \int_{0}^{x}[\alpha(x)-\eta(x)] d x
$$

where $\alpha$ is the Townsend's first coefficient of ionization, $\eta$ is the electron attachment coefficients and $\gamma_{p h}$ is the Townsend's second coefficient of ionization. $\alpha$ and $\eta$ depend on the strength of electric field at the head of avalanche growing withinthe ionization-zone shown in Fig. 5. Expressions of $\alpha$ and $\eta$ as functions of electric field $E$ and air pressure $P$ were reported before [5].

The avalanche continues to grow until it reaches the ionization zone boundary, where $\alpha=\eta$. The number of the emitted photoelectrons $N_{e p h}$, emitted from the stressed electrode during the avalanche growth up to distance $x$ from the stressed electrode,

$$
N_{e p h}=\gamma_{p h} \int_{x_{0}}^{x} \alpha(x) N_{e}(x) g(x) e^{-\mu x} d x
$$

where $g(x)$ is a geometry factor to account for the fact that some photons are not received by the stressed conductor as estimated before [5] and $\mu$ is the coefficient of photon absorption in air [5].

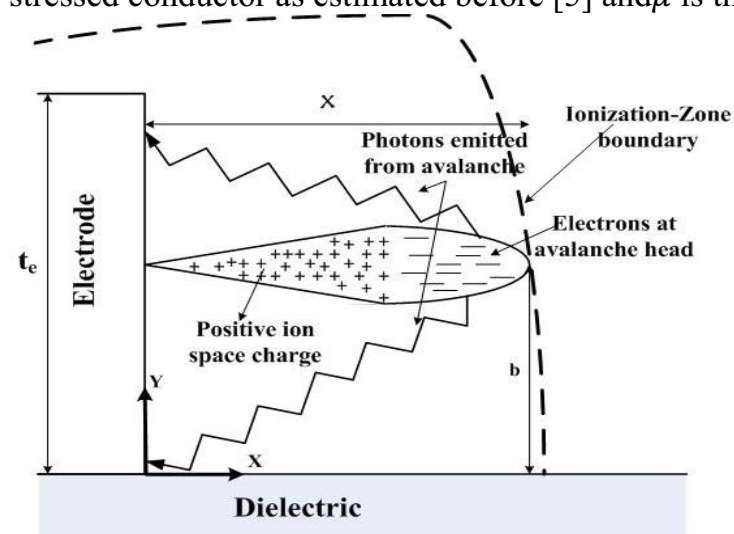

Figure 4. Growth electron avalanche within ionization zone around the stressed electrode edge.

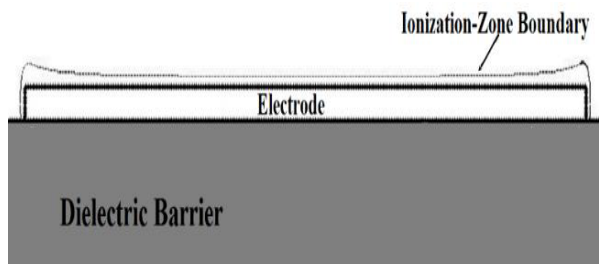

Figure 5. Ionization zone boundary around the stressed electrode in the plane $x-y$.

Therefore, the onset voltage condition is satisfiedwhen the total number of photoelectrons is equal to or more than unity in order to sustain the sliding discharge over the dielectric surface [5].

$$
N_{e p h} \geq 1
$$

The onset voltage does not appear explicitly in the equation. However, the applied voltage effects in the values of $x, \alpha(x)$ and $\eta(x)$ along the growth of the avalanche. The peak value of the applied voltage which satisfies the relation (7) is the onset voltage of sliding discharge (SDBD) over the dielectric barrier.

\section{Results and Discussion}

The width $l_{e}$ and thickness $t_{e}$ are equal $10 \mathrm{~mm}$ and $0.1 \mathrm{~mm}$ respectively. The thickness $t$ lies in the range $2-6 \mathrm{~mm}$. The spacing $S$ is varying over the range $0-20 \mathrm{~mm}$. The relative permittivity $\varepsilon_{r}$ is 
chosenequal to 4 and 8 . With $N_{e}=540$ and $N=2300$, Dirichlet and Neumann conditions are satisfied with acceptable accuracy.

Figure 6 shows the increase of the onset $V_{0}$ of the sliding discharge over the dielectric surface with the increase of the dielectric thickness $t$ for different inter-electrode spacing $S$. For the same values of the spacing $S$ and relative permittivity $\varepsilon_{r}$, the inter electrode capacitance $C$ decreases with the increase of the dielectric barrier thickness $t$ with a subsequent decrease of the electrodes charge at a given applied voltage. This results in a decrease of the electric field in the vicinity of the stressed electrode, i.e. within the ionization-zone surrounding the stressed electrode as shown in Fig. 5. Within this zone, the electron avalanches grow to sustain the sliding discharge over the dielectric surface. To compensate for the decrease of the electric field within the ionization zone, the applied voltage has to increase. This is why the onset voltage $V_{0}$ of the sliding discharge increases with the increase of the thickness of the dielectric barrier as shown in Fig. 6. For a given thickness $t$ value, the onset voltage $V_{0}$ increases with the increase of the spacing $S$ as shown in Fig. 6 . This may be attributed to the fact that the streamlines of the electric field which emanate from the stressed electrode extend over a large surface area of the dielectric barrier. Subsequently, the electric field over the dielectric surface decreases and the applied voltage to sustain the sliding discharge increases with the increase of the spacing $S$. This is why the onset voltage $V_{0}$ increases with the spacing $S$ for the same value of dielectric thickness $t$ as shown in Fig. 6.

Figure 7 shows the increase of the onset voltage $V_{0}$ of sliding discharge over the dielectric surface with the increase of spacing $S$ for different thickness $t$ values of the dielectric barrier. For the same values of thickness $t$ and relative permittivity $\varepsilon_{r}$, the increase of the inter electrode spacing $S$ as stated above makes the streamlines of the electric field emanating from the stressed electrode to extend over a large surface area of the dielectric barrier with a subsequent decrease of the electric field over the dielectric surface. This calls for an increase of the applied voltage to sustain the sliding discharge. This is why the onset voltage $V_{0}$ increases with the increase of spacing as shown in Fig. 7 for the same thickness $t$ and relative permittivity $\varepsilon_{r}$. For a given spacing $S$, the onset voltage $V_{0}$ increases with the dielectric thickness $t$ in agreement withFig. 7 .

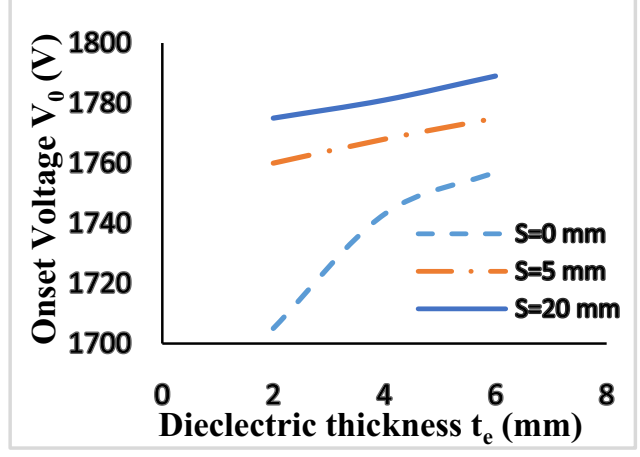

Figure 6. Onset voltage $\left(\boldsymbol{V}_{\mathbf{0}}\right)$ of sliding discharge as influenced by dielectric barrier thickness $(\boldsymbol{t})$ for different spacing $(\mathrm{S})$ values between electrodes, $\left(\varepsilon_{\mathrm{r}}=4, \boldsymbol{t}_{\boldsymbol{e}}=0.1 \mathrm{~mm}\right)$.

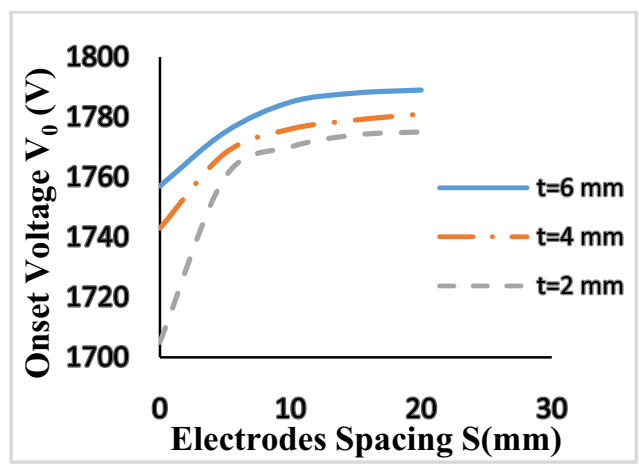

Figure 7. Onset voltage $\left(\boldsymbol{V}_{\mathbf{0}}\right)$ of sliding discharge as influenced by the spacing $(\mathrm{S})$ between the electrodes for different thickness $(\boldsymbol{t})$ values of dielectric barrier, $\left(\varepsilon_{\mathrm{r}}=4, \boldsymbol{t}_{\boldsymbol{e}}=0.1 \mathrm{~mm}\right)$.

Figure 8 shows the increase of the onset voltage $V_{0}$ of the sliding discharge over the dielectric surface with the increase of spacing $S$ for different relative permittivity $\varepsilon_{r}$ values. The increase of $V_{0}$ with the increase of $S$ is explained inFig. 7. For the same values of spacing $S$ and dielectric thicknesst, the onset voltage $V_{0}$ decreases with the increase of the relative permittivity $\varepsilon_{r}$ of the dielectric barrier as shown inFig. 8. This is because the inter electrode capacitance $C$ increases with the increase of dielectric relative permittivity $\varepsilon_{r}$ for constant values of $S$ and $t$ with a subsequent increase of the electrodes chargeat a given applied voltage.This results in an increase of the electric field within the ionization-zone surrounding the stressed electrode. To counter balance the increase of the electric field within the ionization-zone, the applied voltage has to decrease. This is why the onset voltage $V_{0}$ of the 
sliding discharge decreases with the increase of the relative permittivity $\varepsilon_{r}$ as shown in Fig. 8 for the same values of thickness $t$ and spacing $S$.

Figure 9 shows the increase of the onset $V_{0}$ of the sliding discharge with the increase of the electrode thickness $t_{e}$ for different spacing $S$ values. For the same values of the spacing $S$ and relative permittivity $\varepsilon_{r}$, the inter electrode capacitance $C$ decreases with the increase of the thickness $t_{e}$ with a subsequent decrease of the electrodes charge at a given applied voltage. This results in a decrease of the electric field within the ionization-zone surrounding the stressed electrode as shown inFig. 5. To compensate for the decrease of the electric field within the ionization zone, the applied voltage has to increase. This is why the onset voltage $V_{0}$ of the sliding discharge increases with the increase of the electrode thickness as shown inFig. 9.

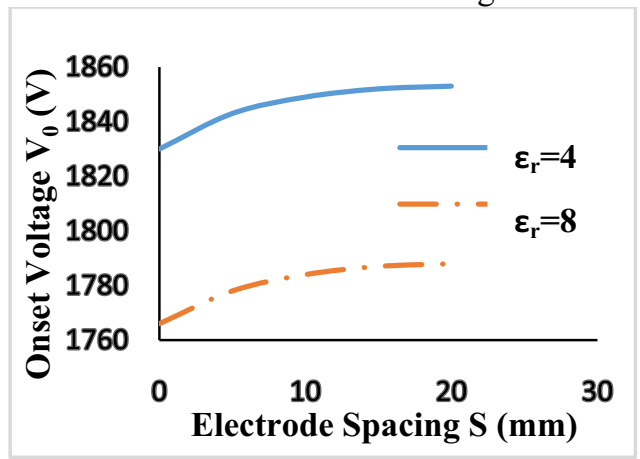

Figure 8. Onset voltage $\left(\boldsymbol{V}_{\mathbf{0}}\right)$ of sliding discharge as influenced by the spacing $(\mathrm{S})$ between the electrodes for different permittivity $\left(\varepsilon_{\mathrm{r}}\right)$ values of dielectric barrier, $\left(\boldsymbol{t}=6 \mathrm{~mm}, \boldsymbol{t}_{\boldsymbol{e}}=0.1 \mathrm{~mm}\right)$.

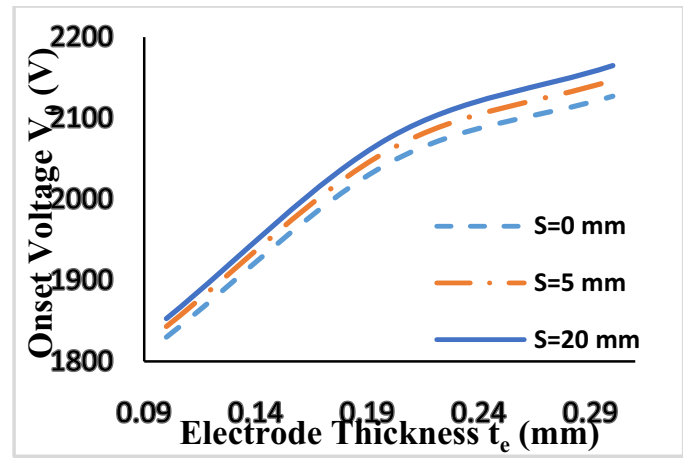

Figure 9. Onset voltage $\left(V_{0}\right)$ of sliding discharge as influenced by electrode thickness $\left(t_{e}\right)$ for different spacing $(\mathrm{S})$ values between electrodes, $\left(\varepsilon_{\mathrm{r}}=4\right.$, $\mathrm{t}=6 \mathrm{~mm})$.

\section{Conclusions}

1. The onset voltage of sliding dielectric barrier discharge driven by AC voltage during negative halfcycle can be calculated by a theoretical method.

2. The calculation of the electric field values in the vicinity of the stressed electrode, which is a prerequisite for onset voltage calculation, is made using the charge simulation method.

3 . The calculated onset voltage decreases with the increase of the relative permittivity $\varepsilon_{r}$, as well as the decresce of $t, S$ and $t_{e}$.

\section{References}

1. Louste, C., Artana, G., Moreau, E. \& Touchard, G. Sliding discharge in air at atmospheric pressure: electrical properties. J. Electrostat.63, 615-620 (2005).

2. Enloe, C. L. et al. Mechanisms and Responses of a Dielectric Barrier Plasma Actuator: Geometric Effects. AIAA J.42, 595-604 (2004).

3. Pons, J., Moreau, E. \& Touchard, G. Asymmetric surface dielectric barrier discharge in air at atmospheric pressure: electrical properties and induced airflow characteristics. J. Phys. D. Appl. Phys.38, 3635-3642 (2005).

4. Soloviev, V. R. Analytical estimation of the thrust generated by a surface dielectric barrier discharge. J. Phys. D. Appl. Phys.45, 025205 (2012).

5. Khalifa, M., M. Abdel-Salam \& M. Abou-Seada. Calculation of negative corona onset votage. IEEE-PES conferance Pap. C 73 160-9, Winter Meet. New York, N.Y, USA (1973). 